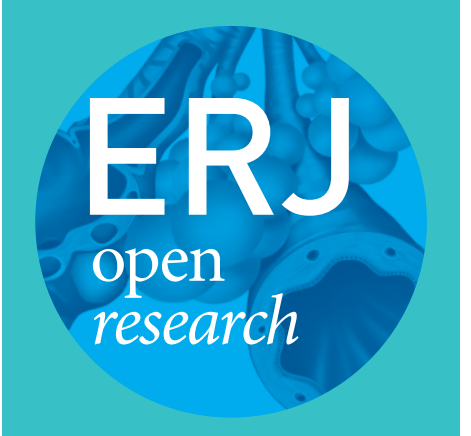

\title{
Mobilising community networks for early identification of tuberculosis and treatment initiation in Cambodia: an evaluation of a seed-and-recruit model
}

\author{
Alvin Kuo Jing Teo $\mathbb{D}^{1}$, Kiesha Prem ${ }^{1,2}$, Sovannary Tuot ${ }^{3}$, Chetra Ork ${ }^{3}$, \\ Sothearith $\mathrm{Eng}^{3}$, Tripti Pande ${ }^{4}$, Monyrath $\mathrm{Chry}^{5}$, Li Yang Hsu ${ }^{1,6}$ and Siyan $\mathrm{Yi}^{1}, 3,7,8$
}

Affiliations: ${ }^{1}$ Saw Swee Hock School of Public Health, National University of Singapore, National University Health System, Singapore. ${ }^{2}$ Dept of Infectious Disease Epidemiology, Faculty of Epidemiology and Population Health, London School of Hygiene and Tropical Medicine, London, UK. ${ }^{3}$ KHANA Centre for Population Health Research, Phnom Penh, Cambodia. ${ }^{4}$ McGill International TB Centre, Montreal, Canada. ${ }^{5}$ Cambodia AntiTuberculosis Association, Phnom Penh, Cambodia. ${ }^{6}$ Yong Loo Lin School of Medicine, National University of Singapore and National University Health System, Singapore. ${ }^{7}$ Center for Global Health Research, Touro University California, Vallejo, CA, USA. ${ }^{8}$ School of Public Health, National Institute of Public Health, Phnom Penh, Cambodia.

Correspondence: Siyan Yi, Saw Swee Hock School of Public Health, National University of Singapore, \#10-01, 12 Science Drive 2, 117549 Singapore. E-mail: ysiyanakhana.org.kh

\section{ABSTRACT}

Background and objectives: The effects of active case finding (ACF) models that mobilise community networks for early identification and treatment of tuberculosis (TB) remain unknown. We investigated and compared the effect of community-based ACF using a seed-and-recruit model with one-off roving ACF and passive case finding (PCF) on the time to treatment initiation and identification of bacteriologically confirmed TB.

Methods: In this retrospective cohort study conducted in 12 operational districts in Cambodia, we assessed relationships between ACF models and: 1) the time to treatment initiation using Cox proportional hazards regression; and 2) the identification of bacteriologically confirmed TB using modified Poisson regression with robust sandwich variance.

Results: We included 728 adults with TB, of whom $36 \%$ were identified via the community-based ACF using a seed-and-recruit model. We found community-based ACF using a seed-and-recruit model was associated with shorter delay to treatment initiation compared to one-off roving ACF (hazard ratio 0.81, 95\% CI 0.68-0.96). Compared to one-off roving ACF and PCF, community-based ACF using a seed-andrecruit model was 45\% (prevalence ratio (PR) $1.45,95 \% \mathrm{CI} 1.19-1.78$ ) and 39\% (PR 1.39, 95\% CI 0.99-1.94) more likely to find and detect bacteriologically confirmed TB, respectively.

Conclusion: Mobilising community networks to find TB cases was associated with early initiation of TB treatment in Cambodia. This approach was more likely to find bacteriologically confirmed TB cases, contributing to the reduction of risk of transmission within the community.

@ERSpublications

A tuberculosis active case finding model that mobilises community networks using a seed-andrecruit approach is associated with early identification and treatment of $T B$, and is also more likely to find bacteriologically confirmed TB cases in Cambodia https://bit.ly/33PWqyR

Cite this article as: Teo AKJ, Prem K, Tuot S, et al. Mobilising community networks for early identification of tuberculosis and treatment initiation in Cambodia: an evaluation of a seed-andrecruit model. ERJ Open Res 2020; 6: 00368-2019 [https://doi.org/10.1183/23120541.00368-2019].

This article has supplementary material available from openres.ersjournals.com

Received: 23 Dec 2019 | Accepted after revision: 23 Feb 2020

Copyright $\odot$ ERS 2020. This article is open access and distributed under the terms of the Creative Commons Attribution Non-Commercial Licence 4.0. 


\section{Introduction}

The ambitious targets set by the World Health Organization (WHO) in the End TB Strategy calls for all people with tuberculosis (TB) to be diagnosed, treated and notified. However, it was estimated that $27 \%$ of the TB cases globally were undiagnosed in 2018 [1]. Cambodia is a high TB burden country, with an estimated incidence of active TB of 302 (95\% CI 169-473) per 100000 population in $2018[1,2]$. In the same year, more than a third of the people with TB in Cambodia were estimated to be undiagnosed [2]. People with TB may have healthcare seeking delays due to limited access to care, financial constraints, geographical barriers, poor community support and/or stigma [3-5]. Prolonged delays to TB diagnosis and treatment, and the burden of undiagnosed cases in the community continue to perpetuate transmission of the infection as TB is an airborne disease [6]. Furthermore, it continues to contribute to the current TB burden and pose a great challenge to TB elimination efforts globally [7, 8]. Additionally, it was estimated that $60 \%$ of Cambodia's population were latently infected with TB in 2014 (i.e. presence of bacteria but not symptoms) [9]. As the population ages, the prevalence of active TB is projected to increase to $33 \%$ in the next decade [10]. It is thus pertinent to reach TB affected individuals, and ensure prompt linkage to diagnosis and effective treatment to break the cycle of TB transmission.

The National Tuberculosis Programme (NTP) in Cambodia has since recalibrated its focus to find the missing cases, and promptly link them to care and treatment [11], especially among key affected populations (KAPs) that are at disproportionate risk of TB [12]. In Cambodia, the KAP for TB was identified as people aged $\geqslant 55$ years, people living with HIV, people with diabetes, prisoners, close contacts of people with bacteriologically confirmed TB and people who abuse drugs [13]. Under the default set-up of the national health system and NTP structure, people with TB who present themselves to the public health facilities will be duly managed and notified to the NTP. This status quo model, termed passive case finding (PCF), relies on people with TB to self-initiate care-seeking, and the capacity of the health facilities to screen and diagnose TB. However, PCF has been shown to be inadequate in finding the missing TB cases in the community [14]. To effectively reach the affected communities, TB active case finding (ACF) activities have been designed and implemented at scale by the NTP and partner civil society organisations in recent years. One attempt at ACF has undertaken a mass screening approach using mobile diagnostic modalities. This is a one-off model and roves from one community to another. A screening session is organised and advertised in the community; target populations (persons aged $\geqslant 55$ years) and other presumptive TB cases are invited to visit the screening site on a specific day. The model has been reported to increase the yield of TB diagnoses two-fold compared with trend-expected notifications and improve treatment outcomes by promptly linking screen-positive individuals to care [15].

The modus operandi of another ACF model focuses on the mobilisation of community networks using a seed-and-recruit approach to find and refer presumptive TB cases to public health facilities for TB screening and diagnosis. The model has been reported to be well-received and deemed feasible by the beneficiaries and key stakeholders [16]. A similar patient-led case-finding model implemented in the Democratic Republic of Congo has shown to increase the yield of TB diagnoses by 42\% [17]. Despite moderate evidence that ACF strategies can detect cases earlier [3, 18, 19], whether ACF results in earlier diagnosis and treatment initiation remains inconclusive primarily due to the variability in the implementation, hence requiring further investigation [20].

The infectivity of $\mathrm{TB}$ is positively correlated with sputum smear status; individuals with sputum smear-positive TB are more infectious than those with smear-negative TB [21]. Therefore, it is pertinent to diagnose and treat people with TB, especially smear-positive cases, to decrease the period of infectivity and halt transmission. This study aimed to investigate the relationships between TB case finding models and the time from the onset of symptoms to $\mathrm{TB}$ treatment initiation and bacteriologically confirmed $\mathrm{TB}$ in Cambodia.

\section{Methods}

\section{Study population}

We conducted a retrospective cohort study among people diagnosed with TB aged $\geqslant 18$ years between February and September 2019 in 12 operational districts (ODs) in 10 provinces across Cambodia. This study was nested within 100 health centres, which were selected with a probability proportional to size (by the total population served by each health centre) sampling without replacement from the sampling frame of 143 health centres in the 12 ODs. Potential study participants were screened by either of the three TB case finding approaches.

\section{TB screening and diagnosis during case finding activities}

For the community-based ACF with a seed-and-recruit model, lay counsellors (TB survivors pre-identified by the project team) acted as focal points at the local health centres to identify seeds in the community 
(other TB survivors and their family members, and other key informants in the community), screen presumptive TB using a symptom assessment questionnaire [12], and train seeds and recruiters to find other presumptive TB in the community in a snowball manner. Presumptive TB cases that exhibited symptoms compatible with TB [12] were further assessed by a nurse practitioner in the health centre for TB workup. The bacteriological status of presumptive TB was determined at the health centres by either smear microscopy (for members of the general population) or GeneXpert MTB/RIF (for TB KAPs). The bacteriological status of study participants who self-presented to the health centre (PCF) was similarly determined. For the one-off roving ACF, the mass screening event was made known to the members of the community, especially among the target population (people aged $\geqslant 55$ years). Individuals who visited the event were screened using a TB symptom questionnaire and chest radiography. The bacteriological status of individuals with an abnormal chest radiograph was determined using GeneXpert MTB/RIF modalities on-site during the screening session. Participants suspected to have extrapulmonary TB underwent further clinical and laboratory examinations at the referral hospital in accordance with the national guidelines [22]. Individuals who tested negative by smear microscopy or GeneXpert MTB/RIF were diagnosed with TB by clinicians based on clinical symptoms and chest radiographic abnormalities [22]. The details of the TB case-finding models are described elsewhere $[16,23]$ and a summary of the interventions are provided in figures S1 and S2. Participants were recruited within 1 month of diagnosis, regardless of bacteriological status, infection site, history of previous TB disease and treatment, HIV status, and drug resistance status. Written and verbal (for illiterate participants) consent were taken for all participants of the study. Those who refused to participate were excluded from the study sample.

\section{Data collection}

Data were collected using a paper-based questionnaire and data collectors were trained prior to data collection. The questionnaire included information on sociodemographic characteristics, smoking and alcohol use, TB symptoms before diagnosis and the date of onset, knowledge and beliefs on TB, stigma-related experiences, and psychological distress. It was piloted among other people with TB and TB survivors. We adapted the World Health Organization TB knowledge, attitude and practices survey [24] to evaluate participants' knowledge of TB symptoms, route of transmission, prevention and treatment of TB. Participants were considered to have poor TB knowledge if they scored the median or below and good TB knowledge otherwise. Using a validated TB stigma scale $[25,26]$ and the General Health Questionnaire (GHQ-12) [27, 28], we measured patients' perspectives toward TB and the psychological distress they experienced before their confirmatory diagnosis, respectively (supplementary materials).

The outcomes of interest in this study were: 1) the time (in days) between the onset of symptoms and TB treatment initiation, defined as total delay; and 2) bacteriologically confirmed TB. The onset of symptoms was self-reported by study participants. Treatment initiation dates and the bacteriological status were extracted from medical records at the health centres and/or case-finding programme databases at the implementing organisations.

As this study was nested within a cohort of people newly diagnosed with TB, identified either through ACF interventions or PCF, who in Cambodia are currently followed up for 6 months from the initiation of TB treatment, no sample size calculation was performed a priori to defining the primary outcome of this study.

\section{Statistical analyses}

Statistical analyses were performed using STATA 14 (StataCorp LP, College Station, TX, USA) and R (R Foundation for Statistical Computing, Vienna, Austria). When appropriate, descriptive statistics were used. Using time-to-event analysis [29, 30], we assessed the relationships between case-finding strategies and total delay as follows. Model 1: community-based ACF with a seed-and-recruit model versus one-off roving ACF; model 2: community-based ACF with a seed-and-recruit model versus PCF. We regarded events to have occurred when TB treatment was initiated. As all participants were diagnosed with TB and initiated on treatment, no data were censored. The statistical significance of the covariates was estimated using the log-rank test and univariate Cox proportional hazard regression. We assessed the association between case-finding strategies and bacteriologically confirmed TB using modified Poisson regression with robust sandwich variance. Epidemiologically relevant variables and other covariates with a p-value $\leqslant 0.1$ in bivariate analyses were considered in the multivariable models. Covariate selection was also guided by directed acyclic graphs of causal inference. Models selection was guided by Akaike information criteria. For Cox regression, we assessed the proportional hazards assumption using Schoenfeld residuals [31] and no violations were observed (model 1: $\mathrm{p}=0.815$; model 2: $\mathrm{p}=0.237$ ). We evaluated the fit of the Cox models using Cox-Snell residuals. Hazard ratios (HRs) $>1$ indicated longer total delays and prevalence ratios $(\mathrm{PR})$ $>1$ indicated higher likelihood of detecting bacteriologically confirmed TB cases. HRs and PRs were 
reported with $95 \%$ confidence intervals and a two-tailed p-value $<0.05$ was considered statistically significant.

\section{Ethics}

This study was approved by the National Ethics Committee for Health Research Cambodia (NECHR ref. 024/NECHR) and the National University of Singapore Institutional Review Board (NUS-IRB ref. H-19-015).

\section{Results}

This cohort included 728 people with TB (table 1) with a mean \pm SD age of $60.1 \pm 13.9$ years. Most participants $(62.2 \%)$ were living in a rural setting. The study population included more males $(53.0 \%)$ than females. Most participants had not attained secondary-level education and most were married at the time of the interview. The median self-reported distance from home to the nearest public health facility was $4 \mathrm{~km}$ (interquartile range 2-6 km). Approximately two-thirds of the participants never smoked cigarettes or drank alcohol and a similar proportion had a good level of knowledge regarding TB. Cough and weight loss were commonly reported symptoms.

\section{Time between the onset of symptoms and TB treatment initiation}

Of the 728 participants, $35.9 \%$ were screened and referred to public health facilities for diagnosis via community-based ACF using a seed-and-recruit model, 54.5\% were screened and diagnosed during the one-off ACF screening event and the remaining 9.6\% self-presented to the health centres (PCF). By design, most participants recruited by community-based ACF using a seed-and-recruit model and PCF were younger than participants screened and diagnosed during the one-off roving ACF screening event. More urban dwellers self-initiated healthcare at the health centres than rural residents (table 1).

Figure 1 compares the time from onset of symptoms to $\mathrm{TB}$ treatment initiation comparing community-based ACF using a seed-and-recruit model and one-off roving ACF. In the bivariate analyses (table 2), community-based ACF using a seed-and-recruit model was associated with shorter total delay (HR 0.70, 95\% CI 0.60-0.82; p<0.001) compared to one-off roving ACF. Adjustments for ODs (urban or rural), age, sex, TB symptoms, psychological distress, TB knowledge, distance from place of residence to public health facilities and TB stigma did not materially affect the effect of community-based ACF using a seed-and-recruit model on total delay (adjusted hazard ratio (aHR) 0.81, 95\% CI 0.68-0.96; p<0.016).

In model 2, we compared the effect of community-based ACF using a seed-and-recruit model with PCF (figure 1 and table 2). After adjusting for similar covariates as model 1, community-based ACF using a seed-and-recruit model was not significantly associated with shorter total delay (aHR 0.93, 95\% CI $0.70-1.23 ; \mathrm{p}=0.610)$.

Across the three TB case finding strategies, we observed no significant heterogeneity in the estimates by age, sex, TB knowledge, urbanicity of ODs and education level of cases detected.

\section{Bacteriologically confirmed TB diagnosis}

We further investigated the effect of case-finding strategies on the detection of bacteriologically confirmed TB. Community-based ACF using a seed-and-recruit model was 45\% (adjusted prevalence ratio (aPR) 1.45, 95\% CI 1.19-1.78; $\mathrm{p}<0.001$ ) and 39\% (aPR 1.39, 95\% CI 0.99-1.94; $\mathrm{p}=0.057$ ) more likely to find and detect bacteriologically confirmed TB compared to one-off roving ACF (model 1) and PCF (model 2), respectively (table 3 ). In the analyses stratified by OD (urban versus rural), community-based ACF using a seed-and-recruit model was significantly more likely to find bacteriologically confirmed TB in urban areas (table 4) but no difference was found in rural areas. Compared to the one-off roving ACF, community-based ACF using a seed-and-recruit model was almost twice more likely to find bacteriologically confirmed TB among females $(p<0.001)$ but no statistically significant trends were observed in males (table 4).

\section{Discussion}

This is the first study that describes and compares the effect of TB case-finding strategies on the time from the onset of symptoms to TB treatment initiation and bacteriologically confirmed TB in Cambodia. In comparison to one-off roving ACF, we observed that the community mobilisation model was associated with a shorter delay to TB treatment initiation. Empirical evidence has shown that community-based, peer-led interventions were effective in identifying HIV cases and reaching new HIV cases in the earlier stages of the infection among marginalised populations such as men who have sex with men [32, 33]. Using a similar mechanism, the community-based ACF using seed-and-recruit model mobilised members of the community to identify presumptive TB cases in their community and promptly link them to care. 
TABLE 1 Characteristics of study participants by case finding strategies and types of tuberculosis (TB)

\begin{tabular}{|c|c|c|c|c|c|c|c|c|}
\hline \multirow[t]{2}{*}{ Characteristics } & \multicolumn{4}{|c|}{ TB case-finding strategies } & \multicolumn{3}{|c|}{ Types of TB } & \multirow[t]{2}{*}{ Total } \\
\hline & $\begin{array}{l}\text { Community-based ACF using } \\
\text { seed-and-recruit model }\end{array}$ & $\begin{array}{l}\text { One-off } \\
\text { roving ACF }\end{array}$ & PCF & p-value & $\begin{array}{c}\text { Smear-negative, } \\
\text { clinician-diagnosed, EРTB }\end{array}$ & $\begin{array}{l}\text { Bacteriologically } \\
\text { confirmed TB }\end{array}$ & p-value & \\
\hline $\begin{array}{l}\text { Time from onset of } \\
\text { symptoms to TB } \\
\text { treatment initiation, } \\
\text { in days }\end{array}$ & $42(24-103)$ & $59(29-144)$ & $38(16-84)$ & $<0.001$ & $56(27-120)$ & $47(23-113)$ & 0.074 & $53(26-118)$ \\
\hline Age, in years & $58(47-67)$ & 65 (55-73) & $56(45-64)$ & $<0.001$ & $63(53-72)$ & $58(49-67)$ & $<0.001$ & $61(52-71)$ \\
\hline $\begin{array}{l}\text { Distance from home to } \\
\text { health facility, } \\
\text { in kilometres }\end{array}$ & $4(2-8)$ & $4(3-6)$ & $4(2-6)$ & 0.119 & $4(2-6)$ & $4(2-7)$ & 0.355 & $4(2-6)$ \\
\hline Age, in years & $56.1 \pm 14.7$ & $63.6 \pm 12.1$ & $54.4 \pm 15.4$ & $<0.001$ & $61.6 \pm 13.6$ & $57.7 \pm 14.2$ & $<0.001$ & $60.1 \pm 13.9$ \\
\hline $\begin{array}{l}\text { Patient perspectives } \\
\text { toward TB }\end{array}$ & $17.8 \pm 5.8$ & $18.6 \pm 4.2$ & $16.4 \pm 5.5$ & 0.001 & $18.1 \pm 4.8$ & $18.1 \pm 5.3$ & 0.890 & $18.1 \pm 5.0$ \\
\hline Operational districts & & & & $<0.001$ & & & 0.520 & \\
\hline Urban & $126(48.3 \%)$ & $95(23.9 \%)$ & $54(77.1 \%)$ & & $164(36.9 \%)$ & $111(39.2 \%)$ & & $275(37.8 \%)$ \\
\hline Rural & $135(51.7 \%)$ & $302(76.1 \%)$ & $16(22.9 \%)$ & & $281(63.1 \%)$ & $172(60.8 \%)$ & & $453(62.2 \%)$ \\
\hline Female & 117 (44.8\%) & $193(48.6 \%)$ & 32 (45.7\%) & 0.620 & 221 (49.7\%) & $121(42.8 \%)$ & 0.069 & $342(47.0 \%)$ \\
\hline Education level" & & & & 0.117 & & & 0.200 & \\
\hline Primary and lower & 215 (82.7\%) & 339 (86.3\%) & $54(77.1 \%)$ & & 377 (85.5\%) & 231 (81.9\%) & & $608(84.1 \%)$ \\
\hline Above primary & $45(17.3 \%)$ & $54(13.7 \%)$ & $16(22.9 \%)$ & & $64(14.5 \%)$ & $51(18.1 \%)$ & & $115(15.9 \%)$ \\
\hline Marital status & & & & 0.001 & & & 0.260 & \\
\hline Never married & 10 (3.8\%) & $16(4.0 \%)$ & $8(11.4 \%)$ & & $21(4.7 \%)$ & $13(4.6 \%)$ & & $34(4.7 \%)$ \\
\hline Currently married & $216(82.8 \%)$ & 294 (74.1\%) & $55(78.6 \%)$ & & 337 (75.7\%) & $228(80.3 \%)$ & & $565(77.6 \%)$ \\
\hline Divorced/widowed & $35(13.4 \%)$ & $87(21.9 \%)$ & $7(10.0 \%)$ & & $87(19.6 \%)$ & $42(14.8 \%)$ & & $130(17.7 \%)$ \\
\hline Ever smoked ${ }^{+}$ & $88(33.7 \%)$ & $103(25.9 \%)$ & $26(37.1 \%)$ & 0.038 & 109 (24.5\%) & $108(38.2 \%)$ & $<0.001$ & $217(29.8 \%)$ \\
\hline Current smokers & $49(18.9 \%)$ & $65(16.4 \%)$ & $19(27.1 \%)$ & 0.098 & $69(15.5 \%)$ & $64(22.8 \%)$ & 0.014 & $133(18.3 \%)$ \\
\hline $\begin{array}{l}\text { Ever consumed } \\
\text { alcohol }{ }^{\pi, s^{5}}\end{array}$ & $92(35.5 \%)$ & $93(23.6 \%)$ & $25(35.7 \%)$ & 0.002 & $111(25.1 \%)$ & $99(35.4 \%)$ & 0.003 & $210(29.0 \%)$ \\
\hline $\begin{array}{l}\text { Presence of other } \\
\text { known medical } \\
\text { conditions }\end{array}$ & $186(71.3 \%)$ & $292(73.6 \%)$ & $51(72.9 \%)$ & 0.812 & $324(72.8 \%)$ & $205(72.4 \%)$ & 0.913 & $529(72.7 \%)$ \\
\hline Cough $^{f}$ & 235 (90.0\%) & 338 (85.1\%) & 66 (94.3\%) & 0.037 & 388 (87.2\%) & 251 (88.7\%) & 0.547 & $639(87.8 \%)$ \\
\hline Haemoptysis ${ }^{f}$ & $62(23.8 \%)$ & $46(11.6 \%)$ & $16(22.9 \%)$ & $<0.001$ & $59(13.3 \%)$ & $65(23.0 \%)$ & 0.001 & $124(17.0 \%)$ \\
\hline Chest painf & $149(57.1 \%)$ & 194 (48.9\%) & $48(68.6 \%)$ & 0.004 & 230 (51.7\%) & $161(56.9 \%)$ & 0.170 & $391(53.7 \%)$ \\
\hline Dyspnoea $^{f}$ & $90(34.5 \%)$ & $120(30.2 \%)$ & $30(42.9 \%)$ & 0.094 & $134(30.1 \%)$ & $106(37.5 \%)$ & 0.040 & $240(33.0 \%)$ \\
\hline Fever $f$ & $153(58.6 \%)$ & $194(48.9 \%)$ & $48(68.6 \%)$ & 0.002 & $250(56.2 \%)$ & $145(51.2 \%)$ & 0.192 & 395 (54.3\%) \\
\hline Chills $^{f}$ & $60(23.0 \%)$ & $56(14.1 \%)$ & $10(14.3 \%)$ & 0.010 & $61(13.7 \%)$ & $65(23.0 \%)$ & 0.001 & $126(17.3 \%)$ \\
\hline Weight loss ${ }^{f}$ & $187(71.7 \%)$ & $245(61.7 \%)$ & 57 (81.4\%) & 0.001 & $303(68.1 \%)$ & $186(65.7 \%)$ & 0.508 & $489(67.2 \%)$ \\
\hline Night sweats ${ }^{f}$ & $128(49.0 \%)$ & $186(46.9 \%)$ & $44(62.9 \%)$ & 0.047 & $219(49.2 \%)$ & $139(49.1 \%)$ & 0.980 & $358(49.2 \%)$ \\
\hline Good TB knowledge & $193(73.9 \%)$ & 295 (74.3\%) & $58(82.9 \%)$ & 0.278 & 345 (77.5\%) & $201(71.0 \%)$ & 0.048 & $546(75.0 \%)$ \\
\hline $\begin{array}{l}\text { Self-perceived risk of } \\
\text { getting TB }\end{array}$ & $163(64.4 \%)$ & $167(46.3 \%)$ & $57(86.4 \%)$ & $<0.001$ & $238(58.3 \%)$ & $149(54.8 \%)$ & 0.359 & $387(56.9 \%)$ \\
\hline
\end{tabular}


Characteristics

TB case-finding strategies

Types of TB

Bacteriologically p-value

\section{Community-based ACF using \\ seed-and-recruit model}

PCF

\section{p-value}

clinicianear-negative, ician-diagnosed, EP
$<0.001$

\section{Total GHQ-12}

$\leqslant 3$

$>3$

\section{Type of TB}

Smear-negative,

clinician-diagnosed

EPTB

Bacteriologically

confirmed TB

Case-finding

\section{strategies}

Community-based

ACF using a

seed-and-recruit

model

One-off roving ACF

PCF
$150(57.5 \%)$

$111(42.5 \%)$

$129(49.4 \%)$

$132(50.6 \%)$

$26(37.1 \%)$
Total

0.618

$\begin{array}{ll}227(51.0 \%) & 139(49.1 \%) \\ 218(49.0 \%) & 144(50.9 \%)\end{array}$

0.618

$366(50.3 \%)$ $362(49.7 \%)$

$445(61.1 \%)$

$283(38.9 \%)$

$<0.001$

$\begin{array}{ccc}129(29.0 \%) & 132(46.6 \%) & 261(35.9 \%) \\ & & \\ 272(61.1 \%) & 125(44.2 \%) & 397(54.5 \%) \\ 44(9.9 \%) & 26(9.2 \%) & 70(9.6 \%)\end{array}$

Data are presented as median (interquartile range), mean \pm SD or $n(\%)$, unless otherwise stated. ACF: active case finding; PCF: passive case finding; EPTB: extrapulmonary tuberculosis; GHQ-12: General Health Questionnaire.": evaluated based on the answers from 12 questions, measured on a Likert scale (0-3), with 0 being strongly disagree and 3 being strongly agree; minimum score is 0 and the maximum score is $36 .{ }^{~ ": ~ e x c l u d i n g ~ m i s s i n g ~ v a l u e s . ~}{ }^{+}$: including current and ex-smokers. ${ }^{\S}$ : participants who had ever consumed alcohol reported frequency of alcohol use that ranged from once a month or less to four times or more per week. $f$ : symptoms prior to TB diagnosis self-reported by study participants. \#\#: evaluated based on the answers to eight questions regarding the characteristics, symptoms, route of transmission, prevention and treatment of TB with a total score of 13 (median 9); respondents were regarded as having poor TB knowledge if they scored the median or below and good TB knowledge if they scored above the median. 17 : evaluated based on the total score of the six negative items; scoring method 0-0-1-1, with $0=$ "less than usual", $0=$ "no more than usual", $1=$ "rather more than usual" or $1=$ "much more than usual". 

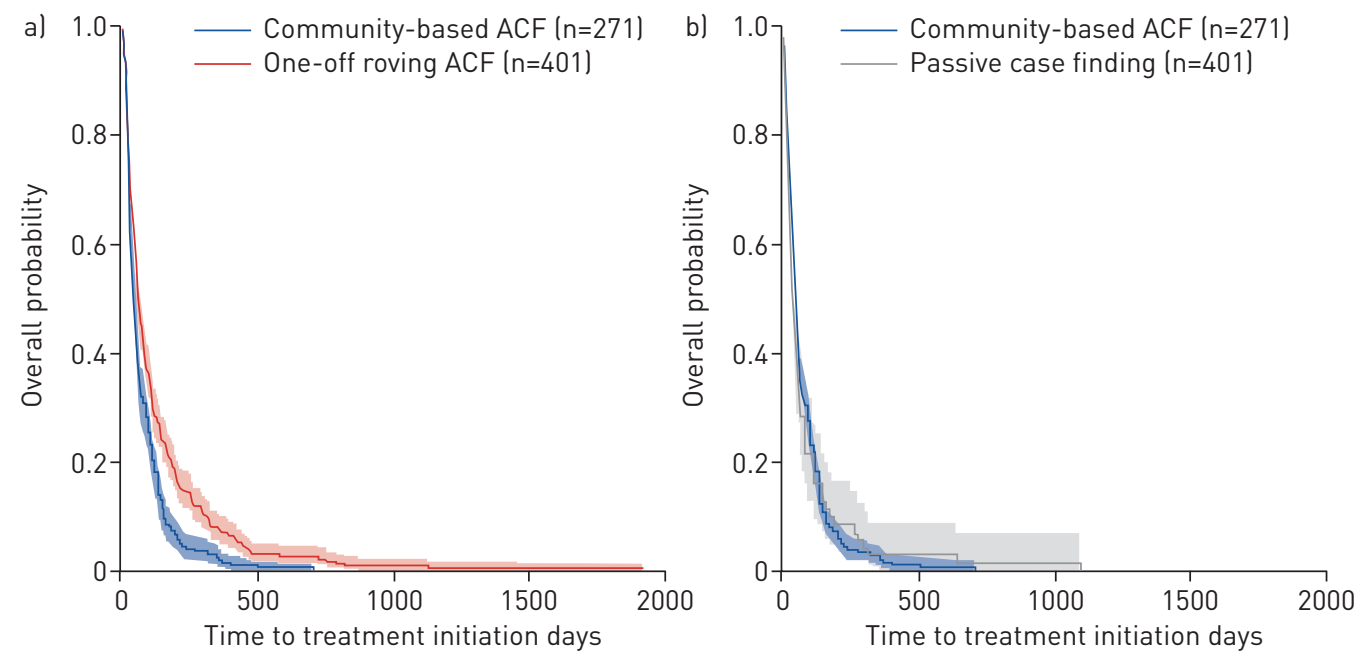

FIGURE 1 Time from the onset of symptoms to tuberculosis (TB) treatment initiation by case-finding strategy. The Kaplan-Meier curves for the time from onset of symptoms to TB treatment initiation comparing: a) community-based active case finding (ACF) using a seed-and-recruit model and one-off roving ACF; and b) community-based ACF using a seed-and-recruit model and passive case finding. The overall probability denotes $P_{\mathrm{r}}(T \geqslant t)$, where $T$ is the time to treatment initiation in days. The solid lines are the product limit estimates and the shaded regions are the $95 \%$ confidence intervals by the complementary log-log method.

Although the spectrum of community mobilisation [34] was not evaluated its entirety, the incorporation of the elements of community participation probably explains the ability of the seeds and recruiters to access KAPs and influence those at risk to seek care early. The empowerment $[35,36]$ that the presumptive $\mathrm{TB}$ cases received from their peers or people living in their community might have enabled them to initiate health-seeking earlier.

There was modest evidence of shorter delay to TB diagnosis and treatment initiation in active screening compared to PCF reported in a recent systematic review [3]. However, in comparison to PCF, our study was unable to discern the effect of community-based ACF using a seed-and-recruit model on the time to treatment initiation. ACF strategies using mobile diagnostic modalities have also been reported to reduce diagnostic delay compared to PCF by improving access to the hard-to-reach populations, such as homeless people and people who abuse drugs [37], thereby lowering the risk of further transmission [38]. However, in our study, we did not observe a similar effect comparing the one-off roving ACF strategy with PCF (table S1 and figure S3). The one-off roving ACF strategy implemented in Cambodia was a targeted approach focused primarily on persons aged $\geqslant 55$ years, with $77 \%$ of the cases belonging to that age group. Therefore, we further stratified the analysis by age (table S2) and we found that, in the older age group (the target population), the one-off roving ACF strategy was associated with shorter total delay compared to PCF, albeit not statistically significant. Although ACF has been shown to be an effective strategy in identifying and linking people with TB to care, a longitudinal study in Peru reported that PCF was found to be equitable in early diagnosis of TB [39]. Notwithstanding that the relationships between the two ACF strategies and PCF were not statistically significant, the effect directions were consistent with evidence that suggested that active screening was inclined to identify TB cases earlier. Hence, we believe that the lack of statistically significant results after accounting for confounders was due to the small sample size, especially in the comparator (PCF).

After adjusting for relevant confounders, we found that the cases identified through community-based ACF using a seed-and-recruit model were more likely to be bacteriologically confirmed TB than cases identified through one-off roving ACF and PCF. The presence of apparent symptoms could have prompted identification and referrals by members of the community who were seeds and recruiters, leading to a higher proportion of bacteriologically confirmed TB cases identified through this model. Previous findings that smear-negative cases were more likely to present with fewer symptoms supported this notion $[40,41]$. We further noted that, in comparison to PCF, cases identified through one-off roving ACF were less likely to be bacteriologically confirmed TB (table S3). This is consistent with a study that evaluated an ACF model using mobile chest radiography, which is similar to the one-off roving ACF model that we assessed [42]. It was suggested that earlier diagnosis might have identified individuals with lower bacillary load and negative sputum smear. However, whether the sputum smear-negative cases were in the subclinical or active TB phase could not be determined using the sample in this study. In a setting 
TABLE 2 Effect of tuberculosis (TB) case-finding strategies on the time (in days) from onset of symptoms to treatment initiation among people with TB in Cambodia

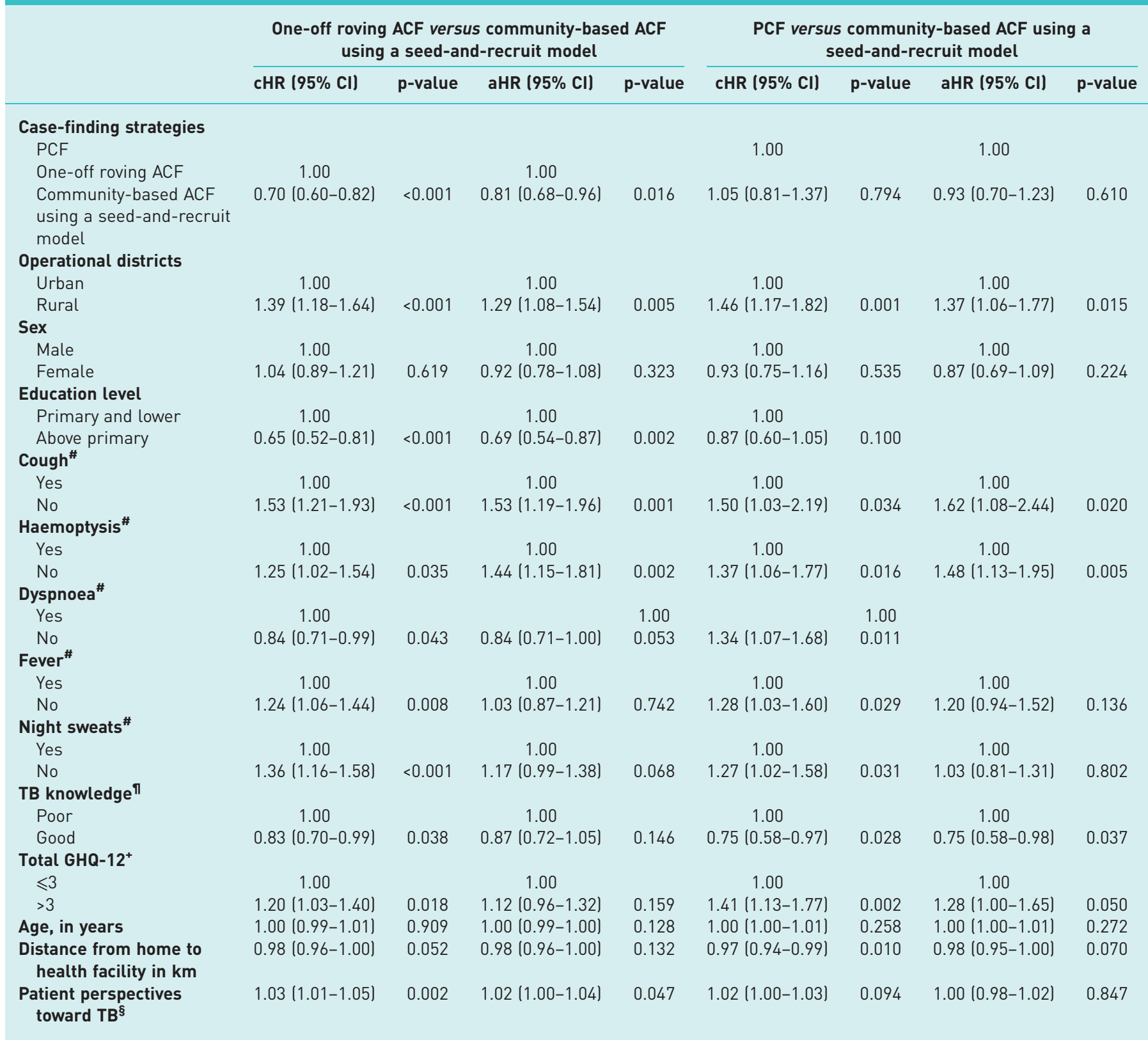

ACF: active case finding; PCF: passive case finding; cHR: crude hazard ratio; aHR: adjusted hazard ratio; GHQ-12: General Health Questionnaire." : symptoms prior to TB diagnosis self-reported by study participants. ${ }^{\text {ๆ: }}$ evaluated based on the answers to eight questions regarding the characteristics, symptoms, route of transmission, prevention and treatment of TB with a total score of 13 (median 9); respondents were regarded as having poor TB knowledge if they scored the median or below and good TB knowledge if they scored above the median. ${ }^{+}$: evaluated based on the total score of the six negative items; scoring method $0-0-1-1$, with $0=$ "less than usual", $0=$ "no more than usual", $1=$ "rather more than usual" or $1=$ "much more than usual". $\S_{\text {: }}$ evaluated based on the answers to 12 questions, measured on a Likert scale (0-3), with 0 being strongly disagree and 3 being strongly agree; minimum score is 0 and the maximum score is 36 .

with $\sim 40 \%$ undiagnosed cases [1], it is crucial to find the missing cases and link them to care promptly, especially bacteriologically confirmed TB and subclinical cases [43], to interrupt transmission.

The strengths of this study include the assessment of total delay in its continuous form to preserve as much information as possible, instead of dichotomising the outcome. Furthermore, no universally acceptable cut-off exists to justify dichotomisation. We nested this study in a routine TB case finding programme supported by the NTP. Therefore, the findings are relevant to real-world practice. In 
TABLE 3 Effect of tuberculosis (TB) case-finding strategies on the detection of bacteriologically confirmed TB in Cambodia

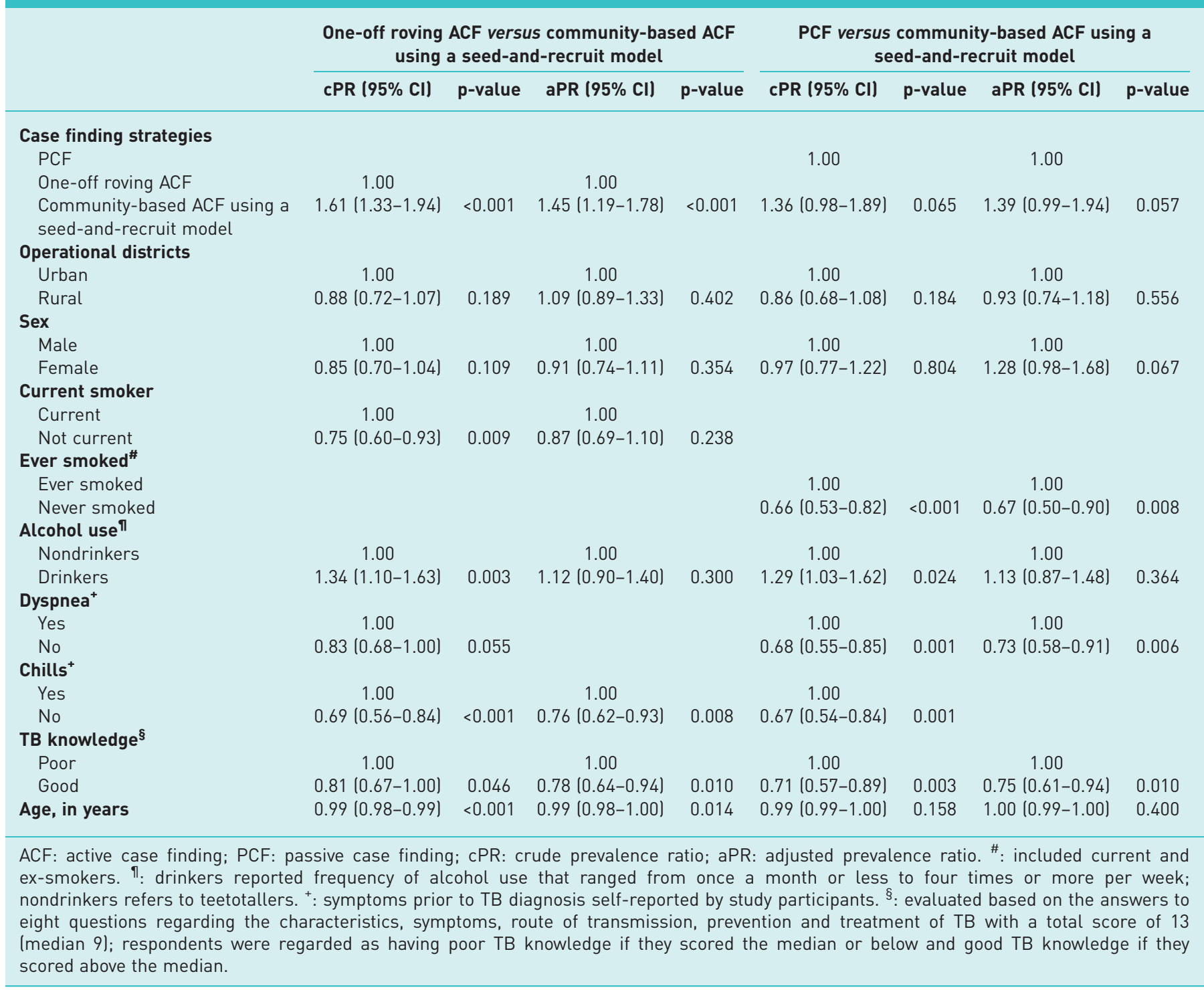

Cambodia, a TB case is diagnosed by using either smear microscopy or GeneXpert MTB/RIF in the community-based ACF using a seed-and-recruit model and PCF. Individuals screened through the one-off roving ACF would undergo a GeneXpert MTB/RIF test for TB diagnosis. Since all the case finding modalities closely followed the national guidelines [22], little within-variation on how cases were diagnosed (i.e. diagnostic tests used) for each case-finding strategy was observed (figure S4). Because the type of diagnostic test was a mediator, it was not adjusted in the regression models, and including it would lead to multicollinearity issues and compromise the true relationship [44].

This study had limitations. Firstly, the dates of onset of symptoms were self-reported by participants and were subjected to recall bias. We strived to minimise recall bias by training data collectors to use significant cultural events and public holidays as prompts to aid recollection of dates. The accuracy of these dates could be further convoluted by people not reporting their symptoms [45] or unable to relate symptoms to TB, hence underreporting the presence of symptoms [46]. No information on the smear grade was collected, which could help inform the stage and infectivity of the disease [19]. In the analyses with PCF as the comparator, small sample size in the PCF group resulted in inadequate statistical power to detect significant differences between the effect sizes. In the context of this study, we were unable to discern our participants' membership of a particular key population other than age. Finally, we cannot rule out residual confounding due to the observational nature of the study design. 


\begin{tabular}{|c|c|c|c|c|}
\hline & \multicolumn{2}{|c|}{ Urban } & \multicolumn{2}{|c|}{ Rural } \\
\hline & $\mathrm{aPR}^{\#}(95 \% \mathrm{Cl})$ & p-value & $\mathrm{aPR}^{\#}(95 \% \mathrm{Cl})$ & $p$-value \\
\hline \multicolumn{5}{|l|}{ Case-finding strategy } \\
\hline One-off roving ACF & 1.00 & & 1.00 & \\
\hline $\begin{array}{l}\text { Community-based } \\
\text { ACF using a } \\
\text { seed-and-recruit } \\
\text { model }\end{array}$ & $2.75(1.75-4.34)$ & $<0.001$ & $1.11(0.86-1.43)$ & 0.417 \\
\hline \multicolumn{5}{|l|}{ Case-finding strategy } \\
\hline PCF & 1.00 & & 1.00 & \\
\hline \multirow{3}{*}{$\begin{array}{l}\text { Community-based } \\
\text { ACF using a } \\
\text { seed-and-recruit } \\
\text { model }\end{array}$} & $1.78(1.18-2.71)$ & 0.007 & $0.79(0.47-1.34)$ & 0.386 \\
\hline & \multicolumn{2}{|c|}{ Male } & \multicolumn{2}{|c|}{ Female } \\
\hline & $\mathrm{aPR}^{11}(95 \% \mathrm{CI})$ & p-value & aPR II $(95 \% \mathrm{CI})$ & p-value \\
\hline \multicolumn{5}{|l|}{ Case-finding strategy } \\
\hline One-off roving ACF & 1.00 & & 1.00 & \\
\hline $\begin{array}{l}\text { Community-based } \\
\text { ACF using a } \\
\text { seed-and-recruit } \\
\text { model }\end{array}$ & $1.12(0.86-1.47)$ & 0.390 & $1.95(1.43-2.66)$ & $<0.001$ \\
\hline \multicolumn{5}{|c|}{$\begin{array}{l}\text { aPR: adjusted prevalence ratio; ACF: active case finding; PCF: passive case finding. " : adjusted for age, } \\
\text { sex, TB symptoms (cough, haemoptysis, fever and night sweats), psychological distress, TB knowledge, } \\
\text { distance from place of residence to public health facilities and TB stigma. ๆ: adjusted for operational } \\
\text { district, age, sex, smoking, alcohol use, TB symptom (chills) and TB knowledge. }\end{array}$} \\
\hline
\end{tabular}

\section{Conclusions}

Mobilising community networks to find TB cases was found to be associated with early identification and initiation of TB treatment in Cambodia. Furthermore, the model was also more likely to find bacteriologically confirmed TB cases and link them to care, contributing to the reduction of risk of transmission in the community. Future systematic investigations are required to evaluate the impact of the different $\mathrm{TB}$ case finding strategies on the yield of $\mathrm{TB}$ diagnoses, the prevalence of $\mathrm{TB}$, reduced transmission and treatment outcomes.

Acknowledgements: We would like to thank the data collectors, field staff and study participants for their contribution to this project. We would like to acknowledge the National Center of Tuberculosis and Leprosy Control Cambodia for their support on this project. The authors thank Marina Smelyanskaya and Lisanne Gerstel for their comments on the paper prior to submission.

Author contributions: A.K.J. Teo, L.Y. Hsu and S. Yi conceptualised and designed the study. A.K.J. Teo, L.Y. Hsu and S. Yi wrote the grant application to obtain funding. A.K.J. Teo, C. Ork, S. Tuot, E. Eng and M. Chry supported project implementation and data collection. A.K.J. Teo and K. Prem supported the design and statistical analysis of the study. A.K.J. Teo, S. Tuot, L.Y. Hsu and S. Yi obtained ethical approval for the project. A.K.J. Teo, K. Prem, C. Ork, S. Eng and M. Chry contributed to the drafting of the manuscript. All authors reviewed and approved the final manuscript.

Support statement: This study was supported by the National University of Singapore Saw Swee Hock School of Public Health Infectious Diseases Program Pilot Grant, National University of Singapore President's Graduate Fellowship and Stop TB Partnership TB REACH Grant. The funders had no role in the design of the study and collection, analysis, and interpretation of data and in writing the manuscript. Funding information for this article has been deposited with the Crossref Funder Registry.

Conflict of interest: None declared.

\section{References}

World Health Organization. Global tuberculosis report 2019. Geneva, World Health Organization, 2019. World Health Organization. TB country profile: Cambodia. Geneva, World Health Organization, 2019. 
3 Kranzer K, Afnan-Holmes $\mathrm{H}$, Tomlin $\mathrm{K}$, et al. The benefits to communities and individuals of screening for active tuberculosis disease: a systematic review. Int J Tuberc Lung Dis 2013; 17: 432-446.

4 Murray EJ, Bond VA, Marais BJ, et al. High levels of vulnerability and anticipated stigma reduce the impetus for tuberculosis diagnosis in Cape Town, South Africa. Health Policy Plan 2013; 28: 410-418.

5 Yaesoubi R, Cohen T. Identifying dynamic tuberculosis case-finding policies for HIV/TB coepidemics. Proc Natl Acad Sci USA 2013; 110: 9457-9462.

6 Golub JE, Bur S, Cronin WA, et al. Delayed tuberculosis diagnosis and tuberculosis transmission. Int J Tuberc Lung Dis 2006; 10: 24-30.

7 Jassal MS, Bishai WR. The epidemiology and challenges to the elimination of global tuberculosis. Clin Infect Dis 2010; 50: S156-S164.

8 Wandwalo E. Make a global priority of finding missing cases of tuberculosis. www.theglobalfund.org/en/blog/ 2017-10-10-make-a-global-priority-of-finding-missing-cases-of-tuberculosis/. Date last accessed: 11 July 2018. Date last updated: 10 October 2017.

9 Houben RMGJ, Dodd PJ. The global burden of latent tuberculosis infection: a re-estimation using mathematical modelling. PLoS Med 2016; 13: e1002152.

10 Prem K, Pheng SH, Teo AKJ, et al. Spatial and temporal projections of the prevalence of active tuberculosis in Cambodia. BMJ Glob Health 2019; 4: e001083.

11 Department of Planning and Health Information. Health strategic plan 2016-2020. Phnom Penh, Department of Planning and Health Information, 2016

12 World Health Organization. Systematic screening for active tuberculosis: an operational guide. Geneva, World Health Organization, 2015.

13 National Center for Tuberculosis and Leprosy Control. Key population assessment in the national tuberculosis response in Cambodia. Phnom Penh, National Center for Tuberculosis and Leprosy Control, 2017.

14 Ho J, Fox GJ, Marais BJ. Passive case finding for tuberculosis is not enough. Int J Mycobacteriol 2016; 5: 374-378.

15 Codlin AJ, Monyrath C, Ky M, et al. Results from a roving, active case finding initiative to improve tuberculosis detection among older people in rural Cambodia using the Xpert MTB/RIF assay and chest X-ray. J Clin Tuberc Other Mycobact Dis 2018; 13: 22-27.

16 Tuot $\mathrm{S}$, Teo AKJ, Cazabon D, et al. Acceptability of active case finding with a seed-and-recruit model to improve tuberculosis case detection and linkage to treatment in Cambodia: a qualitative study. PLoS One 2019; 14: e0210919.

17 André E, Rusumba O, Evans CA, et al. Patient-led active tuberculosis case-finding in the Democratic Republic of the Congo. Bull World Health Organ 2018; 96: 522-530.

18 Shewade HD, Gupta V, Satyanarayana S, et al. Patient characteristics, health seeking and delays among new sputum smear positive TB patients identified through active case finding when compared to passive case finding in India. PLoS One 2019; 14: e0213345.

19 Eang MT, Satha P, Yadav RP, et al. Early detection of tuberculosis through community-based active case finding in Cambodia. BMC Public Health 2012; 12: 469.

20 Prasad BM, Satyanarayana S, Chadha SS, et al. Experience of active tuberculosis case finding in nearly 5 million households in India. Public Health Action 2016; 6: 15-18.

21 Tostmann A, Kik SV, Kalisvaart NA, et al. Tuberculosis transmission by patients with smear-negative pulmonary tuberculosis in a large cohort in the Netherlands. Clin Infect Dis 2008; 47: 1135-1142.

22 National Center for Tuberculosis and Leprosy Control (CENAT). Technical guidelines on tuberculosis control 2nd Edn. Phnom Penh, Ministry of Health, 2016

23 Teo AKJ, Prem K, Evdokimov K, et al. Effect of community active case-finding strategies for detection of tuberculosis in Cambodia: study protocol for a pragmatic cluster randomized controlled trial. Trials 2020; 21: 220.

24 World Health Organization. Advocacy, communication and social mobilization for TB control: a guide to developing knowledge, attitude, and practice surveys. Geneva, World Health Organization, 2008.

25 Van Rie A, Sengupta S, Pungrassami P, et al. Measuring stigma associated with tuberculosis and HIV/AIDS in southern Thailand: exploratory and confirmatory factor analyses of two new scales. Trop Med Int Health 2008; 13: 21-30.

26 Meershoek A, Zwerling A, Daftary A, et al. TB stigma measurement guidance. The Hague, KNCV Tuberculosis Foundation, 2018.

27 Goldberg DP, Gater R, Sartorius N, et al. The validity of two versions of the GHQ in the WHO study of mental illness in general health care. Psychol Med 1997; 27: 191-197.

28 Goldberg D, Williams P. A user's guide to the general health questionnaire. Windsor, NFER-Nelson, 1988

29 Kaplan EL, Meier P. Nonparametric estimation from incomplete observations. J Am Stat Assoc 1958; 53: 457-481.

30 Cox DR. Regression models and life-tables. JR Stat Soc B 1972; 34: 187-202.

31 Schoenfeld D. Chi-squared goodness-of-fit tests for the proportional hazards regression model. Biometrika 1980; 67: $145-153$.

32 Suthar AB, Ford N, Bachanas PJ, et al. Towards universal voluntary HIV testing and counselling: a systematic review and meta-analysis of community-based approaches. PLoS Med 2013; 10: e1001496.

33 Golden MR, Gift TL, Brewer DD, et al. Peer referral for HIV case-finding among men who have sex with men. AIDS 2006; 20: 1961-1968.

34 Khasnabis C, Heinicke Motsch K, Achu K. Community-based rehabilitation: CBR guidelines. Geneva, World Health Organization, 2010.

35 Blanchard AK, Mohan HL, Shahmanesh M, et al. Community mobilization, empowerment and HIV prevention among female sex workers in south India. BMC Public Health 2013; 13: 234.

36 Das A, Friedman J, Kandpal E, et al. Strengthening malaria service delivery through supportive supervision and community mobilization in an endemic Indian setting: an evaluation of nested delivery models. Malar J 2014; 13: 482.

37 Story A, Welfare R, Abubakar I, et al. Targeted mobile digital radiography to reduce diagnostic delay for tuberculosis among hard to reach groups. Int J Tuberc Lung Dis 2008; 12: S143.

38 Den Boon S, Verver S, Lombard CJ, et al. Comparison of symptoms and treatment outcomes between actively and passively detected tuberculosis cases: the additional value of active case finding. Epidemiol Infect 2008; 136 : $1342-1349$

39 Saunders MJ, Tovar MA, Collier D, et al. Active and passive case-finding in tuberculosis-affected households in Peru: a 10-year prospective cohort study. Lancet Infect Dis 2019; 19: 519-528. 
40 Nguyen M-VH, Levy NS, Ahuja SD, et al. Factors associated with sputum culture-negative $v s$ culture-positive diagnosis of pulmonary tuberculosis. JAMA Netw Open 2019; 2: e187617.

41 Campos LC, Rocha MVV, Willers DMC, et al. Characteristics of patients with smear-negative pulmonary tuberculosis (TB) in a region with high TB and HIV prevalence. PLoS One 2016; 11: e0147933.

42 Story A, Aldridge RW, Abubakar I, et al. Active case finding for pulmonary tuberculosis using mobile digital chest radiography: an observational study. Int J Tuberc Lung Dis 2012; 16: 1461-1467.

43 Houben RMGJ, Esmail H, Emery JC, et al. Spotting the old foe-revisiting the case definition for TB. Lancet Respir Med 2019; 7: 199-201.

44 Baron RM, Kenny DA. The moderator-mediator variable distinction in social psychological research: conceptual, strategic, and statistical considerations. J Pers Soc Psychol 1986; 51: 1173-1182.

45 Onozaki I, Law I, Sismanidis C, et al. National tuberculosis prevalence surveys in Asia, 1990-2012: an overview of results and lessons learned. Trop Med Int Health 2015; 20: 1128-1145.

46 Esmail H, Dodd PJ, Houben RMGJ. Tuberculosis transmission during the subclinical period: could unrelated cough play a part? Lancet Respir Med 2018; 6: 244-246. 